\title{
Genome-wide analysis of chalcone synthase (CHS) family from eggplant (Solanum melongena L.) in flavonoid biosynthetic pathway and expression pattern in response to heat stress
}

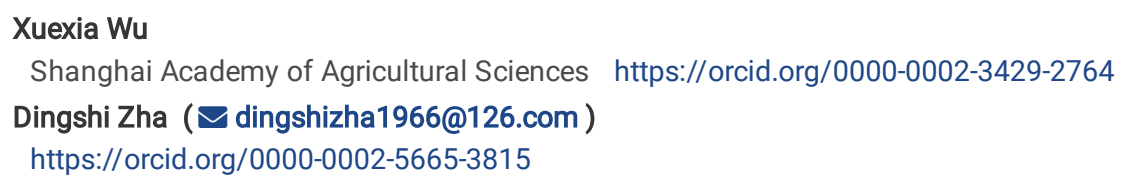

\section{Research article}

Keywords: CHS gene family, Chalcone synthase, Anthocyanin, Heat stress, Expression pattern

Posted Date: November 19th, 2019

DOI: https://doi.org/10.21203/rs.2.17462/v1

License: (c) (i) This work is licensed under a Creative Commons Attribution 4.0 International License. Read Full License 


\section{Abstract}

Background Enzymes of chalcone synthase (CHS) family participate in the synthesis of a series of secondary metabolites in plants, fungi and bacteria. Some of these metabolites have a wide variety of biological functions such as flower pigmentation, protection against UV radiation, pathogen defense, auxin transport and pollen fertility. CHS also showed significant correlation to the accumulation patterns of anthocyanin. The peel color mainly determined by the content of anthocyanin, is a majority economic trait for eggplant affected by heat stress.

Results A total of 7 CHS ( SmCHS1-7) putative genes were identified in genome-wide of eggplant ( S. melongena L . ). The SmCHS genes distribute on 7 scaffolds and were classified into 3 clusters. Phylogenetic relationships analysis showed that $73 \mathrm{CHS}$ genes from 7 Solanaceae species were classified into 10 groups. SmCHS5, SmCHS6 and SmCHS7 were continuously down-regulated under $38^{\circ} \mathrm{C}$ and $45^{\circ} \mathrm{C}$ treatment, while SmCHS 4 was up-regulated under $38^{\circ} \mathrm{C}$ but little change at $45^{\circ} \mathrm{C}$ in peel. Expression profiles of anthocyanin biosynthesis key genes families showed that the PAL, $4 \mathrm{CL}$ and $\mathrm{AN} 11$ genes were mainly expressed in all five tissues. CHI, F3H, F3'5'H, DFR, 3GT and bHLH1 genes were expressed in flower and peel. Under heat stress, 52 key genes expression level were reduced under heat stress. By contrast, expression patterns of eight key genes similar to SmCHS4 up-regulated at the $38^{\circ} \mathrm{C}-3 \mathrm{~h}$.

Conclusions Comparative analysis of putative CHS protein biochemical characteristics, cis -regulatory elements, regulatory network revealed that $\mathrm{SmCHS}$ genes family have conservation gene structure and functional diversification. SmCHS showed two or more expression patterns and execute multiple functions to regulate anthocyanin content. Combined with regulatory networks, it is possible to further understand the regulation mechanism of peel color in eggplant.

\section{Background}

Enzymes of chalcone synthase (CHS) is the member of plants-specific type III ployketide synthase (PKS) [1, 2], catalyses the first committed step of the branch of the phenylpropanoid pathway which leads to the synthesis of flavonoids [3,4]. Flavonoids are well known as a group of plant secondary metabolites that comprise several different classes of compounds such as chalcones, flavones, flavonols isoflavones and anthocyanins. They have a wide variety of biological functions, such as flower pigmentation, protection against UV radiation, pathogen defense, auxin transport and pollen fertility [5-7]. $\mathrm{CHS}$ also showed significant correlation to the accumulation patterns of anthocyanin. After heat treatment, Transcript levels of $\mathrm{CHS}$ decreased in rose flower and in eggplant $[8,9]$.

The product of the CHS reaction is a pivotal precursor for a vast array of secondary metabolites derived from malonyl-CoA and p-coumaroyl-CoA. CHS exists as homodimeric iterative PKS (monomer size of $42-45 \mathrm{kDa}$ ) with two independent active sites that catalyze a series of decarboxylation, condensation, and cyclization reactions $[2,10]$. CHS superfamily share high similarity in their amino acid sequence, which contains the structurally conserved catalytic center consisted of four residues Cys-His-Asn-Phe, and most of the genes contain two exons and one intron [11]. But, the CHS genes family has been not characterized yet in eggplant.

Anthocyanins are plant secondary metabolites and one of the most abundant natural pigments, that are responsible for the characteristic many colors in flowers, fruits and vegetables plant tissues. The anthocyanin biosynthesis pathway has been studied in numerous plant species and most of the genes involved in this process have been identified. The enzyme evolved in anthocyanin biosynthesis are as follows: phenylalanine ammonia lyase (PAL), cinnamate 4-hydroxylase ( $\mathrm{C} 4 \mathrm{H})$, 4-coumarateCoA ligase $(4 \mathrm{CL})$, chalcone synthase $(\mathrm{CHS})$, chalcone isomerase (CHI), flavanone 3-hydroxylase (F3H), flavonoid 3 '-hydroxylase(F3 'H), flavonoid 3 '5 '-hydroxylase (F3 '5 'H), dihydroflavonol4-reductase (DFR), anthocyanidin synthase (ANS), and anthocyanidin 3-O-glucosyltransferase (3GT). Besides, Most transcription factors, such as myeloblastosis (MYB) and basic helix-loop-helix (bHLH), are positive regulators of anthocyanin biosynthesis in vegetative tissues [12-14]. Production of chalcone requires the condensation of one molecule of $p$ coumaroyl-CoA and three malonyl-CoA molecules which is catalyzed by CHS. In conclusion, CHS as the gatekeeper of anthocyanin pathway [15].

Anthocyanins play an important role in plant survival under stressful environmental conditions. high temperatures are known to reduce anthocyanin accumulation and have discoloration effect in many plant tissues. even drastically in colored flowers [8, 16], and the skin of fruits such as grape berries, apples and eggplant $[9,17-20]$.

Eggplant (S. melongena L.) is one of the most important thermophilic vegetable which is produced in many tropical and temperate regions of the world. The optimum growth temperature for eggplant is between 22 and $30^{\circ} \mathrm{C}$. Eggplants subjected to high temperature may lead to stagnation of growth, abortion of flower buds, decrease of pollen viability rate and fruit set, and the peel's color will turn light when the temperature is over $35^{\circ} \mathrm{C}$. High temperature severely reduce the yield and affects the appearance quality of eggplant. However, the molecular mechanism of high temperature stress in eggplant, are poorly understood.

In current study $₫ a l l ~ S m C H S$ family members were identified in eggplant. The comprehensive survey of members was performed including gene structures, The biochemical characteristics of putative CHS protein, promoters cis-elements, phylogenetic relationships among members in other relative species, as well as their expression profiles in various organs/tissues under high temperature stress. The findings of the present study will provide a new start for functional studies on eggplant SmCHS family genes.

\section{Methods}




\section{Plant materials and RNA extraction}

Eggplant cultivar 'Tewangda' is a cold tolerance cultivar with blackish purple skin. It is grow vigorous and have good fruit setting. The fruit shape is $27.6 \mathrm{~cm}$ fruit length, $5.4 \mathrm{~cm}$ transverse diameter and $209 \mathrm{~g}$ single fruit weight on average. The 'Tewangda' fruit has good commercial property and good transportation resistance. 'Tewangda' were grown at the same growth stage were randomly selected. These plants were grown 144 days after sowing, then put inside incubators set at $27^{\circ} \mathrm{C}(\mathrm{CK}), 38^{\circ} \mathrm{C}$ or $45^{\circ} \mathrm{C}$ for 3 or 6 hours (three plants per treatment). For each treatment, the tissue samples of root, stem, leaf, flower and peel were obtained and immediately frozen in liquid nitrogen, and stored at $-80^{\circ} \mathrm{C}$ for RNA extraction and other analyses. All plant materials examined in this study were obtained from Shanghai Academy of Agricultural Sciences. The total RNA was extracted from each tissue sample by mirVana miRNA Isolation Kit (Ambion) following the manufacturer's protocol. The extracted total RNA was stored at $-80^{\circ} \mathrm{C}$. RNA integrity was evaluated using the Agilent 2100 Bioanalyzer (Agilent Technologies, Santa Clara, CA, USA).

\section{Identification of the CHS family members in eggplant genome}

The whole protein sequence of Solanum melongena L. (eggplant) were obtained from the Eggplant Genome DataBase (http://eggplant.kazusa.or.jp) [21], and those of Solanum tuberosum L. (potato, http://solanaceae.plantbiology.msu.edu/pgsc_download.shtml) [22], Solanum lycopersicum (tomato, https://solgenomics.net/organism/Solanum_lycopersicum/genome) [23], Solanum penellii (wild tomato, https://www.plabipd.de/project_spenn/start.ep) [24], Capsicum annuum L. (pepper, http://peppergenome.snu.ac.kr) [25], Petunia axillaris (https://solgenomics.net/organism/Petunia_axillaris/genome) [26], Petunia inflate (https://solgenomics.net/organism/Petunia_inflata/genome) [26], Nicotiana tabacum (common tobacco, https://www.ncbi.nlm.nih.gov/nuccore/AYMY00000000) [27]. The profiles of CHS (PF00195 and PF02797) was download from Pfam protein family database (http://pfam.xfam.org/) and these profile sequence were used as query to perform BLASTP search against the protein sequence data of all the species which mentioned above with a maximum E-value of $1 \times 10^{-3}$, respectively [28]. To further verify the exact copy number of CHS and remove redundant sequences, the Pfam database and Genome websites were also searched using "chalcone synthase" as keyword. All CHS sequences were submitted to EXPASy (https://web.expasy.org/protparam/) to calculate the number of amino acid, molecular weight and theoretical isoelectric point $(\mathrm{pl})$.

\section{Structure characterization}

The locations and intron numbers of CHS were acquired through the genome website. All of the acquired protein sequences wreath first aligned by ClustalX software with the default parameters [29]. An unrooted maximum-likelihood phylogenetic tree was constructed using MEGA6 software with bootstrap test of 1000 times [30]. The MEME program (Version 5.0.5, http://meme-suite.org/tools/meme) was used to identified the conserved motif of the CHS sequences with the following parameters, any number of repetitions, maximum of 10 misfits and optimum motif width of 6-200 amino acid residues. The WoLF PSORT program was used to predict the subcellular localization information of CHS proteins (https://www.genscript.com/wolfpsort.html) [31].

\section{Analysis of cis-acting element in SmCHS}

The upstream sequences $(2 \mathrm{~kb})$ of the $\mathrm{SmCHS}$ coding sequences in eggplant were retrieved from the genome sequence, and then submitted to PlantCARE (http://bioinformatics.psb.ugent.be/webtools/plantcare/html/) to identify regulatory elements [32].

\section{Phylogenetic analysis of $\mathrm{CHS}$ genes}

The full-length protein sequences of all eight species in Solanaceae were used for phylogenetic analysis. All of the protein sequences were first aligned by ClustalX software with the default parameters [29]. The phylogenetic tree was generated with MEGA6 software with bootstrap test of 1000 times. The final tree was viewed and modified in Evolview software [33]. The CHS genes were classified into different groups according to the topology of phylogenetic tree.

\section{Construction of the mRNA regulatory network}

RNA-seq results come from our lab [34]. The significant differentially expressed genes (Fold change $\geq 2$ and $p$ value $\otimes 0.05$ ) were used to calculate pearson correlation coefficient between $\mathrm{CHS}$ genes and others. The TBtools program was used to elucidate the Gene Ontology (GO) functional classification for the mRNAs which with the correlation coefficient more than 0.9 [35]. The top 5 regulatory mRNAs which annotated by GO enrichment for the genes associated with anthocyanin biosynthesis were collected to construct the regulatory network. The network was visualized using Cytoscape [36].

\section{qRT-PCR analysis}


The first-strand cDNA was synthetised from $1 \mu \mathrm{g}$ of 5 tissues (root, stem, leaf, flower and peel) total RNA using Prime Script RT Reagent Kit (Takara, Dalian, China). The qRT-PCR reactions were performedin 96-well plates using the ABI 7500 fast Real-Time PCR system (Applied Biosystems, USA) with the QuantiFast SYBR Green PCR Kit (Qiagen, Duesseldorf, Germany). The qRT-PCR parameters were as follows: $95^{\circ} \mathrm{C}$ for 5 min, then 45 cycles of $95{ }^{\circ} \mathrm{C}$ for $10 \mathrm{~s}, 60^{\circ} \mathrm{C}$ for $10 \mathrm{~s}$, and $72^{\circ} \mathrm{C}$ for $10 \mathrm{~s}$. The relative mRNA expression levels were calculated using the $2^{-} \triangle \triangle \mathrm{CT}$ method [37]. PGK(JX154676) was used as an internal control to normalize the data. The primer sequences are listed in Additional file3 Table S1.

\section{Results}

Identification of CHS genes and sequence analysis in Solanaceae species

A total of $7 \mathrm{CHS}(\mathrm{SmCHS1-7)}$ genes in eggplant were identified after verified by protein sequences analysis and Blast search by using eggplant genome annotation database (Additional file1 Table S1a). The length of SmCHS protein ranged from 327 to 396 amino acids (Table 1). The molecular weights of SmCHS were between $35.2 \mathrm{kDa}$ and $43.7 \mathrm{kDa}$. The theoretical pl value of SmCHS ranged from 5.59 to 7.04 . In addition, $66 \mathrm{CHS}$ genes were characterized from other 7 Solanaceae species. The subfamily numbers of CHS genes were ranged from 6 (Solanum penellii) to 13 (Petunia axillaris) (Table 1, Additional file1 Table S1b-h). The molecular weights of CHS for other 7 Solanaceae species range from 17.3 to 47.4 , length of protein ranged from 156 to 431 amino acids, the theoretical pl value ranged from to 5.1 to 8.47 (Additional file2 Table S1a-g). The average of number of amino acid, molecular weight and theoretical pl were calculated, and then as a data set for each specie. The correlation coefficient between the above data were all more than 0.99. This suggests that CHS genes are conservative in Solanaceae species.

\section{Structure and conserved motif analysis of SmCHS}

The $7 \mathrm{SmCHS}$ genes distribute on 7 scaffolds. To better understand the evolution of SmCHS genes, an unrooted maximum-likelihood tree was constructed based on the $7 \mathrm{SmCHS}$ protein sequences, and the SmCHS were classified into 3 clusters (i, ii and iii) (Figure 1). Among the SmCHS genes, only one SmCHS7 had three exons, the others had two exons (Figure 1) based on available information from the genome annotation. These results suggest the potential biological function diversities of the SmCHS genes in eggplant.

To understand the function diversification of $\mathrm{SmCHS}$, the conserved motifs of these 7 protein sequences were identified by MEME program, 10 conserved motifs were detected in eggplant (Figure 2, Table 2). For all the 7 eggplant SmCHS proteins, Motif 1 and Motif 2 exist in all of them, Motif 3 is only absent in SmCHS5, Motif 4 and Motif 5 are only absent in SmCHS1. The N-terminal domain (PF00195) of CHS protein contained Motif 1 and the combination of Motif 3, 4, 6, 7 and 9. The C-terminal domain (PF02797) of CHS protein contained Motif 2 and the combination of Motif 5, 8 and 10. Therefore, the motif configuration of the SmCHS reflects the conservation and diversity of the CHS family. To further investigate subcellular localization information of SmCHS proteins, WoLF PSORT program was used to predict the localization of SmCHS protein [31]. SmCHS7 was predicted to localize in nuclear, SmCHS4 and SmCHS6 were predicted to localize in chloroplast. The others SmCHSproteins were predicted to localize in cytoplasmic. The different compositions of the domains and subcellular localization may indicate functional diversity.

\section{Stress-related cis-elements in SmCHS promoters}

To further study the potential regulatory mechanisms of SmCHS during abiotic stress responses, the $2 \mathrm{~kb}$ upstream sequences from the translation start sites of SmCHS were used to identify the ciselements (Figure 3B). Results showed that all SmCHS had common upstream promoter elements, including TATA-box and CAAT-box which occurred more than 100 times, and therefore, these sequences were presumed to be the promoter sequences (Figure 3 A). The elicitor response (ERE) and myeloblastosis (MYB) occurred more than 10 times in the SmCHS upstream sequences. Research showed that increase of CHS activity causes a high accumulation flavonoid level that inhibit polar auxin transport [15, 38, 39]. Two cis-acting elements (ABRE, involved in abscisic acid responsiveness; AuxRR, involved in auxin responsiveness) were found in the upstream regions. MYB and myelocytomatosis (MYC) binding site have also been identified which may greatly influence plant stress tolerance. These results showed that SmCHS is activated by a wide range of environmental and developmental stimuli, and there are many complex ways to regulate SmCHS activity in eggplant.

\section{Phylogenetic analysis of $\mathrm{CHS}$ genes in Solanaceae}

To analyze the evolutionary relationships of CHS genes in Solanaceae, an unrooted phylogenetic tree was constructed using full length amino acid sequences. All the 73 CHS genes were classified into 10 groups (Figure 4, Table 3), the number of CHS gene groups ranged from two to eleven. The 7 SmCHS genes were categorized into 6 groups (group $\nabla, \nabla, \nabla, \nabla, \nabla$ and $\nabla$ ), and the group $\nabla$ contained SmCHS1 and $S m C H S 2$. The group $\nabla, \nabla, \nabla$ and $\nabla$ exist in all eight species, the group $\otimes, \nabla$ and $\otimes$ were absent in Solanum melongena L., Solanum penellii, Solanum lycopersicum and Solanum tuberosum L.. And the group $\mathbb{Q}$ is absent in Capsicum annuum L., Nicotiana tabacum, Petunia inflate and Petunia axillaries (Table 3). Those results suggested that the CHS genes were conservative, but small variations exist among the eight species in Solanaceae, and also showed that the SmCHS1, SmCHS2 and SmCHS3 were more conservative than the gene SmCHS4 according the phylogenetic tree. 
Using the RNA-seq data, a heatmap of 96 anthocyanin biosynthesis key genes (PAL, C4H, 4CL, CHS, CHI, F3H/F3'H, F3'5'H, DFR, ANS, 3GT, MYB1, MYB2, bHLH1, AN11, MADS1) was established under heat stress (Figure 5). The expression of anthocyanidin synthase (ANS) and MYB2 were not identified during this sampling period. For seven SmCHS genes, three of them (SmCHS5, SmCHS6, SmCHS7) were not identified, the other four SmCHS were divided into two group according their expression patterns. Three of those four SmCHS genes (SmCHS5, SmCHS6, SmCHS7) were continuously downregulated under $38^{\circ} \mathrm{C}$ and $45^{\circ} \mathrm{C}$ treatment compared with the CK. However, SmCHS4 was up-regulated under $38^{\circ} \mathrm{C}$ but little change at $45^{\circ} \mathrm{C}$ in peel. And these phenomena have also been observed in some others key gene families associated to anthocyanin biosynthesis.

\section{The mRNA regulatory network associated with anthocyanin biosynthesis in eggplant}

A total of 4928 mRNAs correlation coefficient were more than 0.9. all of these mRNAs were functionality categorized in GO database. The top 20 GO enrichment results of biological processes were showed in Table 4 . The function was involved in

regulation of biological process (G0:0050789), regulation of cellular metabolic process (G0:0031323) and regulation of gene expression (G0:0010468) were collected and filtered to construct regulatory network. Totally, 67 anthocyanin biosynthesis key genes and 146 regulatory mRNAs were included in this regulatory network (Additional file4 Figure S1). Those GO enrichment results suggest that the anthocyanin biosynthesis pathway maybe regulated by a wide range of environmental and developmental stimuli.

\section{Expression pattern of anthocyanin biosynthesis key genes in different tissues under heat stress}

Using the qRT-PCR data, a heatmap of 20 anthocyanin biosynthesis key genes was established in different tissues under heat stress (Figure 6). The qRTPCR results showed a high consistency with RNA-seq data, which suggested that RNA-seq data were credible. Most of the CHS genes were expressed in peel, and were low expressed in other tissues. The PAL, 4CL and AN11 genes were mainly expressed in all five tissues. The CHI, F3H, F3'5'H, DFR, 3GT and bHLH1 genes were expressed in flower and peel. The MADS1 were expressed in stem, leaf, flower and peel.

\section{Discussion}

It is now known that the $\mathrm{CHS}$ gene family plays a significant role during the growth and development of plants. In many species, multigene family of CHS are identified. For example, Six CHS genes have been described in Turnip [40]. In maize, 14 complete $C H S$ genes have been identified [41]. Total of $27 \mathrm{CHS}$ genes were found in rice [42]. These studies showed that $\mathrm{CHS}$ members were divided into two or more subclasses according to phylogenetic analysis. Generally, genes grouped into the same subclasses shared similar evolutionary features, and also obtained same expression pattern. In our study, the identified sequences showed the high level of coding sequences similarity (above $90 \%$ ). The $\mathrm{SmCHS}$ were classified into three clusters based on the result of maximum-likelihood tree. Under heat stress $\llbracket$ cluster i were continuously down-regulation, cluster ii were up-regulated 4 times under $38^{\circ} \mathrm{C}$ compared with $\mathrm{CK}$ in peel, while cluster iii were not detected the expression in eggplant most tissues. Under $35^{\circ} \mathrm{C}$, previous studies showed that $\mathrm{SmCHS1}$ and SmCHS3 (Sme2.5_01077.1_g00016.1, Sme2.5_13923.1_g00001.1) were down-regulated in peels of eggplant (Lv et al. 2019), which are in agreement with our results. These result suggest that functional diversification of SmCHS.

Flavonoids have numerous functions and contribute to pigments, signaling molecules, protectants against biotic and abiotic Stresses. The flavonoid biosynthetic pathway is one of the most intensively investigated pathways for applied biological and genetic processes, as well as for understanding gene regulation, characterization of transposable elements and production of agronomically stress-tolerant plants, and natural dietary antioxidants. biosynthesis of anthocyanins responds to environmental stressors such as light, nutrient depletion, and temperature change. The peel color determined by the content of anthocyanin, is a majority economic trait for eggplant, which are modulated by the genes in flavonoid biosynthesis pathway. Compared with the other tissues, SmMYB1 and all anthocyanin biosynthetic key genes (SmCHS, SmCHI, SmF3H, SmDFR) except SmPAL were dramatically upregulated in the fruit skin of the purple cultivar [43]. The full lengh cDNA of $S m C H S, S m C H I, S m F 3^{\prime} 5^{\prime} H, S m D F R$, were isolated from eggplant by Jiang. These genes have highest expression levels in peels except for SmF3H which was detected in stems [44]. The expression profiles of these key gene families under heat stress were investigated in our study. The PAL, 4CL and AN11 genes were mainly expressed in all five tissues. The $\mathrm{CHI}, \mathrm{F} 3 \mathrm{H}, \mathrm{F} 3^{\prime} 5^{\prime} \mathrm{H}, \mathrm{DFR}, 3 \mathrm{GT}$ and bHLH1 genes were expressed in flower and peel, inferred that these genes respond at late-stage of anthocyanin pathway directly regulate the color of fruit skin and flower.

Heat stress reduced the anthocyanin content and the enzyme activities of CHS, DFR, ANS and 3GT/UFGT in eggplant peel, and strengthened the activity of PAL [45] [34]. When the temperature is over $35^{\circ} \mathrm{C}$, the eggplant will be dehydrated and shrink, and peel's color will turn light. CHS is a key enzyme of the flavonoid biosynthesis pathway. Most of the genes associated with flavonoid biosynthesis were down-regulated under heat stress. In this study, the genes of the flavonoid biosynthesis pathway showed tissue-specific and genes express in different phases and tend to change with the time (Figure 6). According to the RNA-seq results of 96 anthocyanin biosynthesis key genes in eggplant peel, $\mathrm{SmCHS4}$ showed the highest expression level at the $38^{\circ} \mathrm{C}-$ 3h along with eight other genes (Sme2.5_03336.1_g00008.1_PAL, Sme2.5_00041.1_g00017.1_4CL, Sme2.5_00283.1_g00002.1_smCHS4,

Sme2.5_00298.1_g00002.1_F3H, Sme2.5_02066.1_g00012.1_F3H, Sme2.5_04260.1_g00001.1_F3H, Sme2.5_15970.1_g00001.1_F3H,

Sme2.5_00670.1_g00012.1_DFR, Sme2.5_00747.1_g00013.1_AN11) (Figure 7).Particularly, Sme2.5_03336.1_g00008.1_PAL expression level under $38^{\circ} \mathrm{C}$ doubled while down-regulated in $45^{\circ} \mathrm{C}$ compared with CK; Sme2.5_00670.1_g00012.1_DFR Sme2.5_00747.1_g00013.1_AN11 expression level

Page 5/15 
increased 3-4 fold and $7-10$ fold under $38^{\circ} \mathrm{C}$, respectively. In addition, 52 genes expression level were reduced under heat stress which were similar to Lv's results [9][9][9][9], while 35 genes expression were not identified. Those results suggested that some anthocyanin biosynthesis key genes contribute to protect the eggplant from the damage of heat stress. Moreover, these genes families exhibited two or more expression patterns and execute multiple genetic functions to regulate anthocyanin content. Combined with regulatory networks, it is possible to further understand the regulation mechanism of peel color in eggplant.

\section{Conclusions}

In this study, a genome-wide analysis about SmCHS genes family in eggplant was performed. The CHS protein biochemical characteristics, phylogenetic relationships, gene structures, cis-regulatory elements, regulatory network and functional predictions of the family members were examined. It is revealed that SmCHS genes family have conservation gene structure and functional diversification. CHS play important roles to anthocyanin biosynthesis pathway, exhibited two or more expression patterns and execute multiple functions to regulate anthocyanin content in eggplant peel under heat stress. This work will make eggplant for further research of functions, regulation and evolution of the CHS family.

\section{Declarations}

\section{Abbreviations}

CHS: Chalcone synthase; PAL: phenylalanine ammonia lyase, $\mathrm{C} 4 \mathrm{H}$ : cinnamate 4-hydroxylase; 4CL: 4-coumarateCoA ligase; $\mathrm{CHI}$ : chalcone isomerase; F3H: flavanone 3-hydroxylase, F3 'H: flavonoid 3 '-hydroxylase, F3 '5 'H: flavonoid 3 ' 5 '-hydroxylase; DFR: dihydroflavonol4-reductase; ANS: anthocyanidin synthase; 3GT: anthocyanidin 3-0-glucosyltransferase; MYB: myeloblastosis; bHLH: basic helix-loop-helix; ERE: elicitor response; MYC: myelocytomatosis.

\section{Acknowledgments}

This work was supported by Agricultural Committee Basic Project (Shanghai Agricultural word (2015) No 6-2-3), the National Key Technology R\&D Program during the 13th Five-Year Plan Period (2017YFD0101904) and China Agriculture Research System (Grant No. CARS-25). The funding bodies didn't play a role in the design of the study and collection, analysis, and interpretation of data and in writing the manuscript.

\section{Author contribution}

DZ put forward the research, ZZ and AD carried out the preparation and treatment of test materials. XL and SJ designed and performed the experiment. $\mathrm{XW}$ and SZ analyzed the data and wrote the manuscript. XW revised the article. All authors read and approved the final manuscript.

\section{Ethics approval and consent to participate}

Not applicable

\section{Consent for publication}

Not applicable

\section{Competing interest}

All the authors agreed the content of the manuscript and had no competing interest.

\section{Availability of data and materials}

All of the datasets supporting the results of this article are included within the article and its Additional files.

\section{References}

1.Schröder J: A family of plant-specific polyketide synthases: facts and predictions. Trends Plant Sci. 1997, 2(10):373-378.

2.Austin MB, Noel JP: The chalcone synthase superfamily of type III polyketide synthases. Nat. Prod. Rep. 2003, 20(1):79-110. 
3.Winkel-Shirley B: It takes a garden. How work on diverse plant species has contributed to an understanding of flavonoid metabolism. Plant Physiol. 2001, 127(4):1399-1404.

4.Pang Y, Shen G, Wu W, Liu X, Lin J, Tan F, Sun X, Tang K: Characterization and expression of chalcone synthase gene from Ginkgo biloba. Plant Sci. $2005,168(6): 1525-1531$.

5.Winkel-Shirley B: Biosynthesis of flavonoids and effects of stress. Curr. Opin. Plant Biol. 2002, 5(3):218-223.

6.Buer CS, Imin N, Djordjevic MA: Flavonoids: new roles for old molecules. J. Integr. Plant Biol. 2010, 52(1):98-111.

7.Falcone FM, Rius SP, Casati P: Flavonoids: biosynthesis, biological functions, and biotechnological applications. Front. Plant Sci. 2012, 3.222.

8.Dela G, Or E, Ovadia R, Nissim-Levi A, Weiss D, Oren-Shamir M: Changes in anthocyanin concentration and composition in 'Jaguar'rose flowers due to transient high-temperature conditions. Plant Sci. 2003, 164(3):333-340.

9.Lv LL, Feng XF, Li W, Li K: High temperature reduces peel color in eggplant (Solanum melongena) as revealed by RNA-seq analysis. Genome 2019, 62(7):503-512.

10.Tropf S, Kärcher B, Schröder G, Schröder J: Reaction Mechanisms of Homodimeric Plant Polyketide Synthases (Stilbene and Chalcone Synthase). A single active site for the condensing reaction is sufficient for synthesis of stilbenes, chalcones, and 6'-deoxychalcones. J. Biol. Chem. 1995,

270(14):7922-7928.

11.Ferrer J, Jez JM, Bowman ME, Dixon RA, Noel JP: Structure of chalcone synthase and the molecular basis of plant polyketide biosynthesis. Nat. Struct. Mol. Biol. 1999, 6(8):775.

12.Zhang F, Gonzalez A, Zhao M, Payne CT, Lloyd A: A network of redundant bHLH proteins functions in all TTG1-dependent pathways of Arabidopsis. Development 2003, 130(20):4859-4869.

13.Teng S, Keurentjes J, Bentsink L, Koornneef M, Smeekens S: Sucrose-specific induction of anthocyanin biosynthesis in Arabidopsis requires the MYB75/PAP1 gene. Plant Physiol. 2005, 139(4):1840-1852.

14.Gonzalez A, Zhao M, Leavitt JM, Lloyd AM: Regulation of the anthocyanin biosynthetic pathway by the TTG1/bHLH/Myb transcriptional complex in Arabidopsis seedlings. The Plant Journal 2008, 53(5):814-827.

15.Dao T, Linthorst H, Verpoorte R: Chalcone synthase and its functions in plant resistance. Phytochem. Rev. 2011, $10(3): 397$.

16.Nozaki K, Takamura T, Fukai S: Effects of high temperature on flower colour and anthocyanin content in pink flower genotypes of greenhouse chrysanthemum (Chrysanthemum morifolium Ramat.). The Journal of Horticultural Science and Biotechnology 2006, 81(4):728-734.

17.Mori K, Goto-Yamamoto N, Kitayama M, Hashizume K: Loss of anthocyanins in red-wine grape under high temperature. J. Exp. Bot. 2007, 58(8):1935-1945.

18.Lin-Wang K, Micheletti D, Palmer J, Volz R, Lozano L, Espley R, Hellens RP, Chagne D, Rowan DD, Troggio M et al: High temperature reduces apple fruit colour via modulation of the anthocyanin regulatory complex. Plant Cell Environ. 2011, 34(7):1176-1190.

19.Movahed N, Pastore C, Cellini A, Allegro G, Valentini G, Zenoni S, Cavallini E, D Incà E, Tornielli GB, Filippetti I: The grapevine VviPrx31 peroxidase as a candidate gene involved in anthocyanin degradation in ripening berries under high temperature. J. Plant Res. 2016, 129(3):513-526.

20.Pastore C, Dal Santo S, Zenoni S, Movahed N, Allegro G, Valentini G, Filippetti I, Tornielli GB: Whole plant temperature manipulation affects flavonoid metabolism and the transcriptome of grapevine berries. Front. Plant Sci. 2017, 8:929.

21. Hideki H, Kenta S, Koji M, Tsukasa N, Satomi N, Akio O, Hirotaka Y, Shusei S, Sachiko I, Satoshi T: Draft genome sequence of eggplant (Solanum melongena L.): the representative Solanum species indigenous to the old world. DNA Res. 2014, 21(6):649-660.

22.Consortium PGS: Genome sequence and analysis of the tuber crop potato. Nature 2011, 475(7355):189.

23.Consortium TTG: The tomato genome sequence provides insights into fleshy fruit evolution. Nature 2012, 485(7400):635.

24.Schmidt MH, Vogel A, Denton AK, Istace B, Wormit A, van de Geest H, Bolger ME, Alseekh S, Maß J, Pfaff C: De novo assembly of a new Solanum pennellii accession using nanopore sequencing. The Plant Cell 2017, 29(10):2336-2348.

25.Kim S, Park M, Yeom S, Kim Y, Lee JM, Lee H, Seo E, Choi J, Cheong K, Kim K: Genome sequence of the hot pepper provides insights into the evolution of pungency in Capsicum species. Nat. Genet. 2014, 46(3):270. 
26.Bombarely A, Moser M, Amrad A, Bao M, Bapaume L, Barry CS, Bliek M, Boersma MR, Borghi L, Bruggmann R: Insight into the evolution of the Solanaceae from the parental genomes of Petunia hybrida. Nature plants 2016, 2(6):16074.

27. Sierro N, Battey JN, Ouadi S, Bakaher N, Bovet L, Willig A, Goepfert S, Peitsch MC, Ivanov NV: The tobacco genome sequence and its comparison with those of tomato and potato. Nat. Commun. 2014, 5:3833.

28.Boratyn GM, Camacho C, Cooper PS, Coulouris G, Fong A, Ma N, Madden TL, Matten WT, McGinnis SD, Merezhuk Y: BLAST: a more efficient report with usability improvements. Nucleic Acids Res. 2013, 41(W1):W29-W33.

29.Thompson JD, Gibson TJ, Plewniak F, Jeanmougin F, Higgins DG: The CLUSTAL_X windows interface: flexible strategies for multiple sequence alignment aided by quality analysis tools. Nucleic Acids Res. 1997, 25(24):4876-4882.

30.Tamura K, Stecher G, Peterson D, Filipski A, Kumar S: MEGA6: molecular evolutionary genetics analysis version 6.0. Mol. Biol. Evol. 2013, 30(12):2725-2729.

31.Horton P, Park K, Obayashi T, Fujita N, Harada H, Adams-Collier CJ, Nakai K: WoLF PSORT: protein localization predictor. Nucleic Acids Res. 2007, 35(suppl_2):W585-W587.

32.Lescot M, Déhais P, Thijs G, Marchal K, Moreau Y, Van de Peer Y, Rouzé P, Rombauts S: PlantCARE, a database of plant cis-acting regulatory elements and a portal to tools for in silico analysis of promoter sequences. Nucleic Acids Res. 2002, 30(1):325-327.

33.Subramanian B, Gao S, Lercher MJ, Hu S, Chen W: Evolview v3: a webserver for visualization, annotation, and management of phylogenetic trees. Nucleic Acids Res. 2019.

34.Zhang S, Zhang A, Wu X, Zhu Z, Yang Z, Zhu Y, Zha D: Transcriptome analysis revealed expression of genes related to anthocyanin biosynthesis in eggplant (Solanum melongena L.) under high-temperature stress. BMC Plant Biol. 2019, 19(1):1-13.

35.Chen C, Xia R, Chen H, He Y: TBtools, a Toolkit for Biologists integrating various biological data handling tools with a user-friendly interface. BioRxiv 2018:289660.

36.Smoot ME, Ono K, Ruscheinski J, Wang P, Ideker T: Cytoscape 2.8: new features for data integration and network visualization. Bioinformatics 2010, 27(3):431-432.

37.Livak KJ, Schmittgen TD: Analysis of relative gene expression data using real-time quantitative PCR and the $2-\Delta \Delta^{C T}$ method. Methods 2001 , 25(4):402-408.

38.Jacobs M, Rubery PH: Naturally occurring auxin transport regulators. Science 1988, 241(4863):346-349.

39.Brown DE, Rashotte AM, Murphy AS, Normanly J, Tague BW, Peer WA, Taiz L, Muday GK: Flavonoids act as negative regulators of auxin transport in vivo in Arabidopsis. Plant Physiol. 2001, 126(2):524-535.

40.Zhou B, Wang Y, Zhan Y, Li Y, Kawabata S: Chalcone synthase family genes have redundant roles in anthocyanin biosynthesis and in response to blue/UV-A light in turnip (Brassica rapa; Brassicaceae). Am. J. Bot. 2013, 100(12):2458-2467.

41.Han Y, Ding T, Su B, Jiang H: Genome-wide identification, characterization and expression analysis of the chalcone synthase family in maize. Int. J. Mol. Sci. 2016, 17(2):161.

42.Han Y, Cao Y, Jiang H, Ding T: Genome-wide dissection of the chalcone synthase gene family in Oryza sativa. Mol. Breeding 2017, $37(10): 119$.

43.Zhang Y, Hu Z, Chu G, Huang C, Tian S, Zhao Z, Chen G: Anthocyanin accumulation and molecular analysis of anthocyanin biosynthesis-associated genes in eggplant (Solanum melongena L.). J. Agr. Food Chem. 2014, 62(13):2906-2912.

44.Jiang M, Liu Y, Ren L, Lian H, Chen H: Molecular cloning and characterization of anthocyanin biosynthesis genes in eggplant (Solanum melongena L.). Acta Physiol. Plant. 2016, 38(7):163.

45.Wu X, Zhang A, Zhu Z, Yao J, Zha D, Li X: Effects of High $\square$ temperature Stress on Active Oxygen Metabolism, Anthocyanin Content and Its Main Synthases in Eggplant Peel. Acta Agriculturae Jiangxi, China 2018, 30(06):1-5.

\section{Tables}

Table 1. Feature of SmCHS genes identified in eggplant. 


\begin{tabular}{lllll}
\hline Gene Name & Gene ID & Number of amino acids & Molecular weight (kDa) & Theoretical pI \\
\hline SmCHS1 & Sme2.5_01077.1_g00016.1 & 333 & 36.4 & 6.61 \\
SmCHS2 & Sme2.5_02154.1_g00001.1 & 389 & 42.7 & 5.97 \\
SmCHS3 & Sme2.5_13923.1_g00001.1 & 389 & 42.5 & 7.04 \\
SmCHS4 & Sme2.5_00283.1_g00002.1 & 392 & 43.1 & 6.14 \\
SmCHS5 & Sme2.5_01039.1_g00002.1 & 327 & 35.2 & 5.59 \\
SmCHS6 & Sme2.5_00346.1_g00019.1 & 396 & 43.7 & 6.16 \\
SmCHS7 & Sme2.5_05261.1_g00004.1 & 383 & 42.6 & 5.67 \\
\hline
\end{tabular}

Table 2 List of the putative motifs of CHS proteins

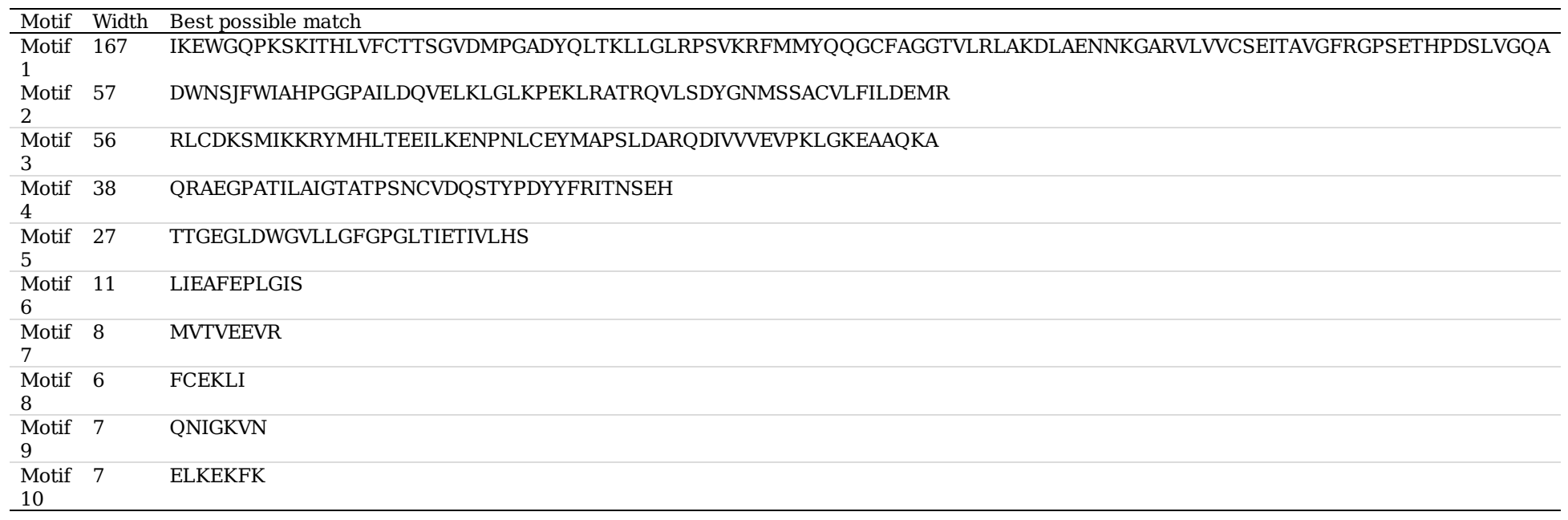

Table 3. The distribution of $C H S$ genes in phylogenetic tree.

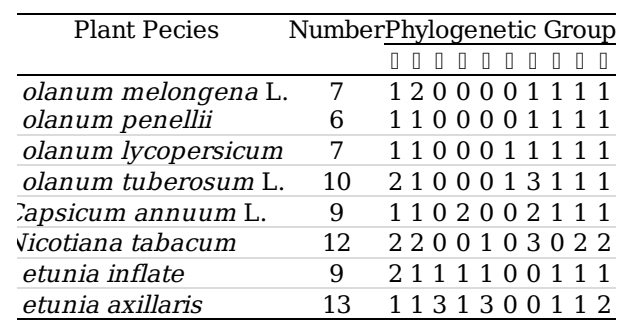

Table 4. The top 20 GO enrichment results of biological processes. 


\begin{tabular}{lll}
\hline GO term & GO ID & P value \\
\hline cellular biosynthetic process & GO:0044249 & 0 \\
cellular nitrogen compound biosynthetic process & GO:0044271 & 0 \\
\hline cellular response to chemical stimulus & $\mathrm{GO}: 0070887$ & 0 \\
\hline cellular response to stress & $\mathrm{GO}: 0033554$ & 0 \\
\hline regulation of biological process & $\mathrm{GO}: 0050789$ & 0 \\
\hline regulation of cellular macromolecule biosynthetic process & $\mathrm{GO}: 2000112$ & 0 \\
\hline developmental process & $\mathrm{GO}: 0032502$ & 0 \\
\hline regulation of RNA biosynthetic process & $\mathrm{GO}: 2001141$ & 0 \\
\hline regulation of cellular metabolic process & $\mathrm{GO}: 0031323$ & 0 \\
\hline cellular component organization & $\mathrm{GO}: 0016043$ & 0 \\
\hline response to organic substance & $\mathrm{GO}: 0010033$ & $1.11 \mathrm{E}-16$ \\
\hline protein metabolic process & $\mathrm{GO}: 0019538$ & $1.11 \mathrm{E}-16$ \\
\hline regulation of nitrogen compound metabolic process & $\mathrm{GO}: 0051171$ & $1.11 \mathrm{E}-16$ \\
\hline regulation of gene expression & $\mathrm{GO}: 0010468$ & $1.11 \mathrm{E}-16$ \\
\hline response to stimulus & $\mathrm{GO}: 0050896$ & $1.11 \mathrm{E}-16$ \\
\hline regulation of nucleobase-containing compound metabolic process & $\mathrm{GO}: 0019219$ & $1.11 \mathrm{E}-16$ \\
\hline response to chemical & $\mathrm{GO}: 0042221$ & $1.11 \mathrm{E}-16$ \\
\hline cell communication & $\mathrm{GO}: 0007154$ & $1.11 \mathrm{E}-16$ \\
\hline response to stress & $\mathrm{GO}: 0006950$ & $1.11 \mathrm{E}-16$ \\
\hline oxoacid metabolic process & $\mathrm{GO}: 0043436$ & $2.22 \mathrm{E}-16$ \\
\hline
\end{tabular}

Figures

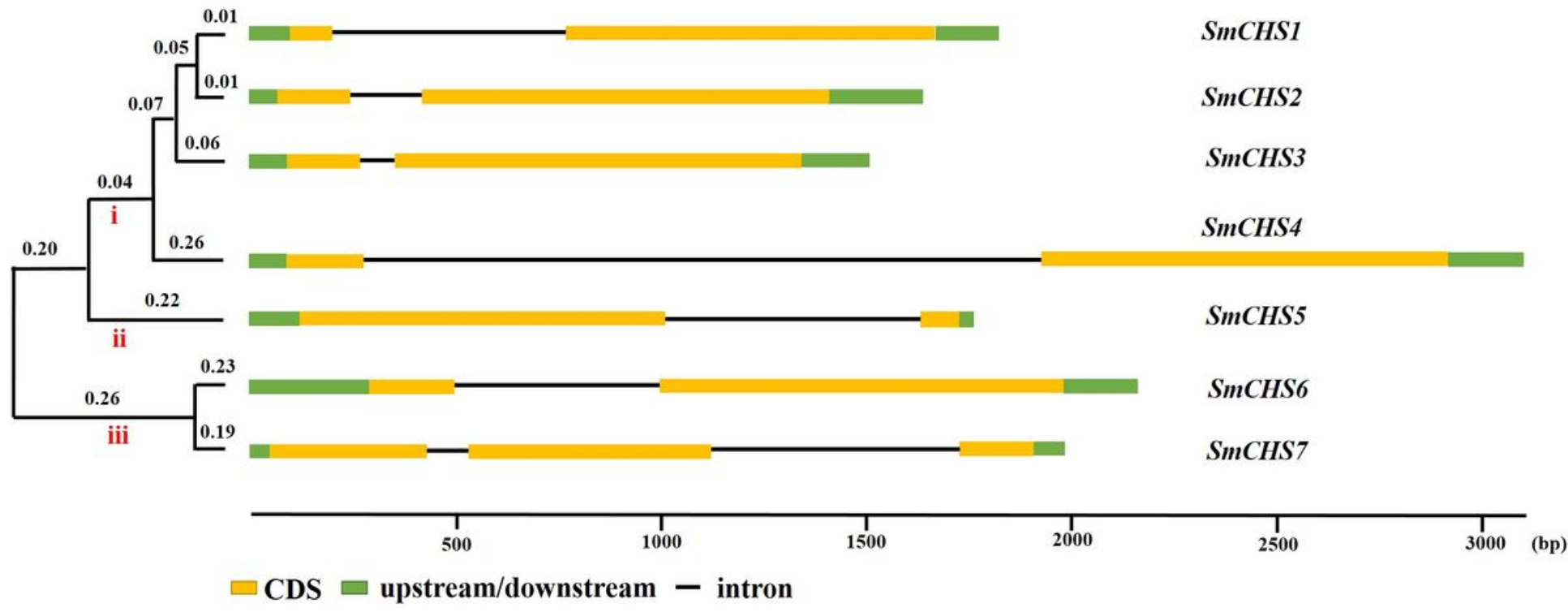

Figure 1

Phylogenetic relationship and gene structure analysis of SmCHS genes. The phylogenetic tree was on the left of the figure, which showed that SmCHS were classified into three clusters (i, ii and iii). The exon/intron organization of SmCHS was on the right of the figure. For SmCHS genes organization, yellow boxes represent exons, black lines represent the intron, and green boxes are indicated in. upstream/downstream region. The length of exon and intron are drawn to scale. 


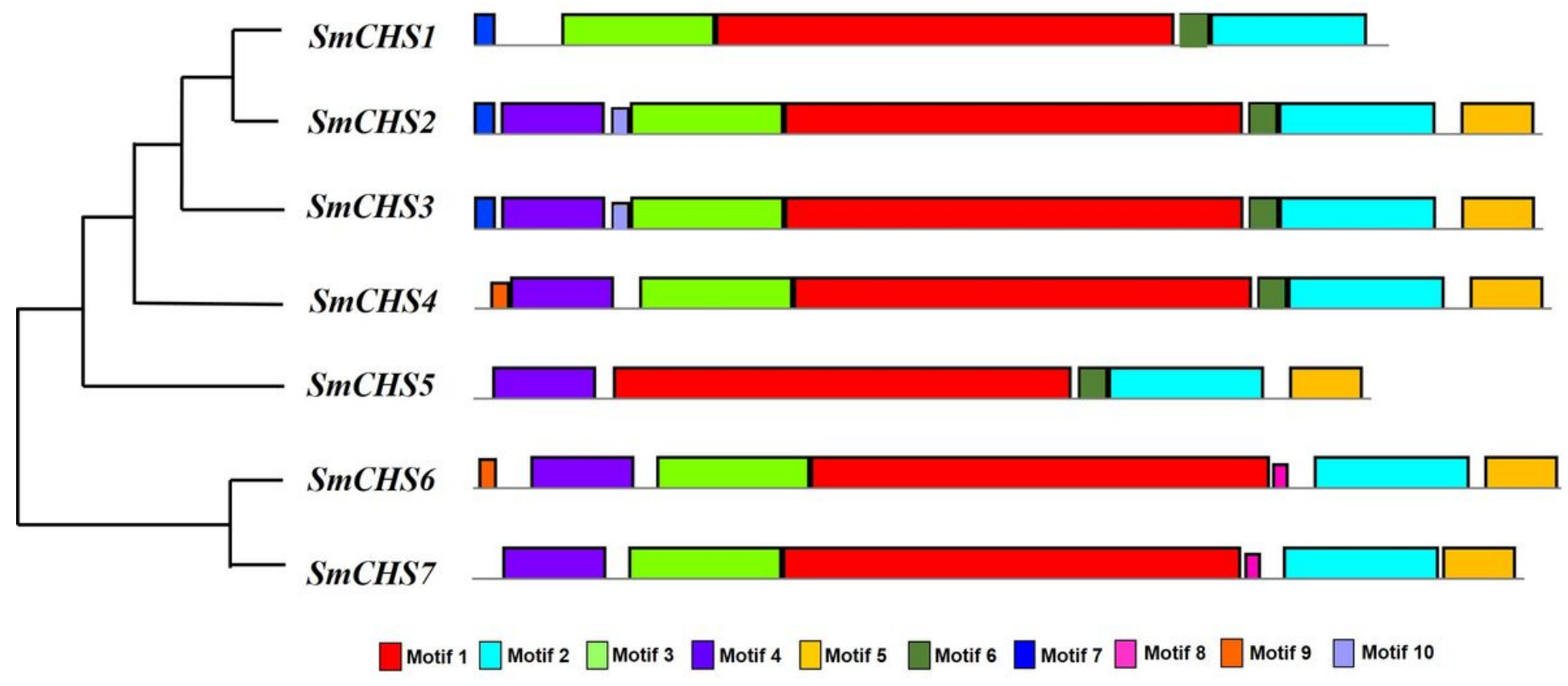

Figure 2

Conserved motifs across all CHS proteins in eggplant. Ten conserved motifs are indicated in different colored boxes.

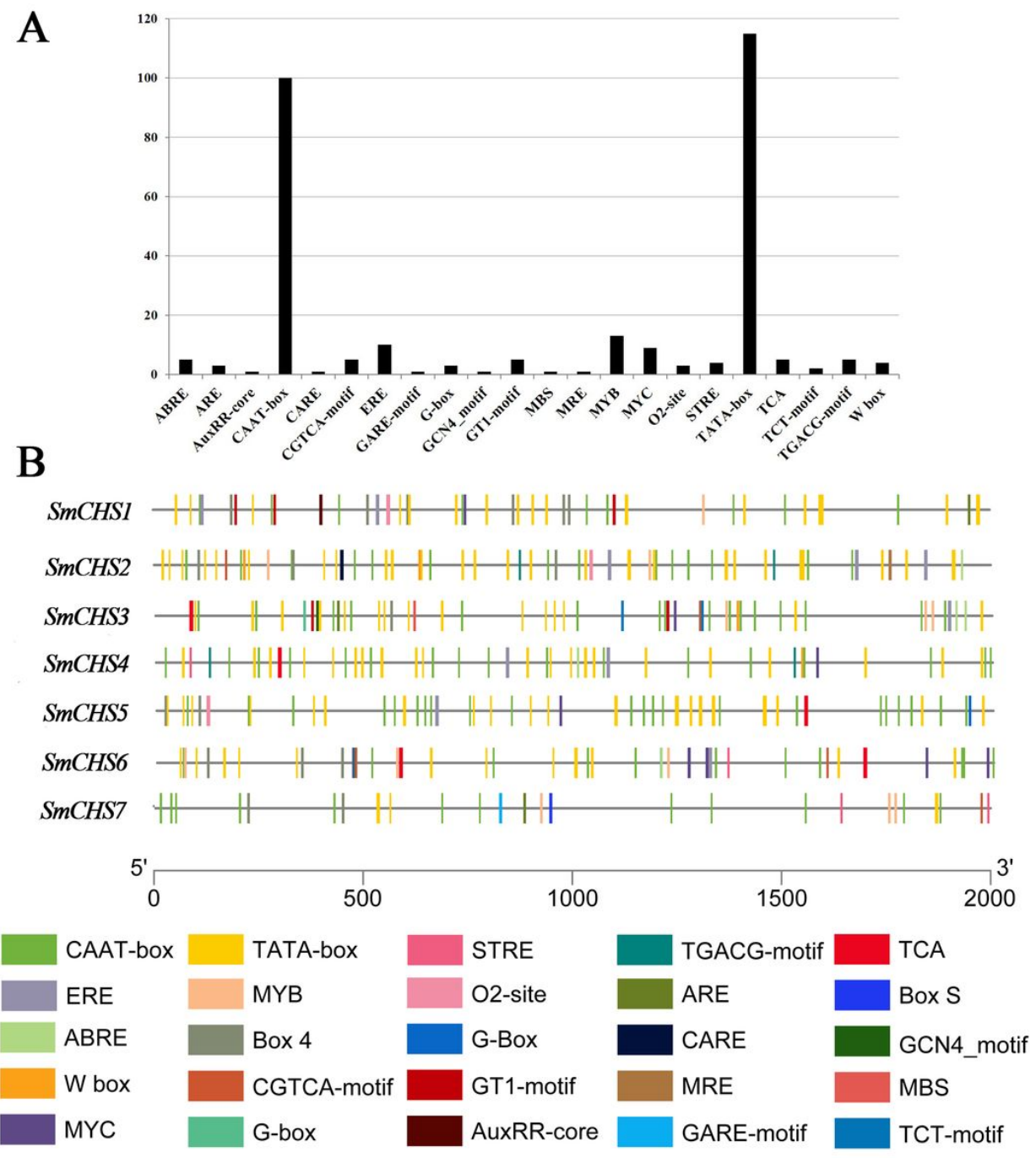

Figure 3 
Cis-elements in CHS family genes promoters. (A) Frequency of cis-element occurrence in upstream sequences. (B) Predicted cis-elements in CHS genes promoters. Scale bar indicates the length of promoters.

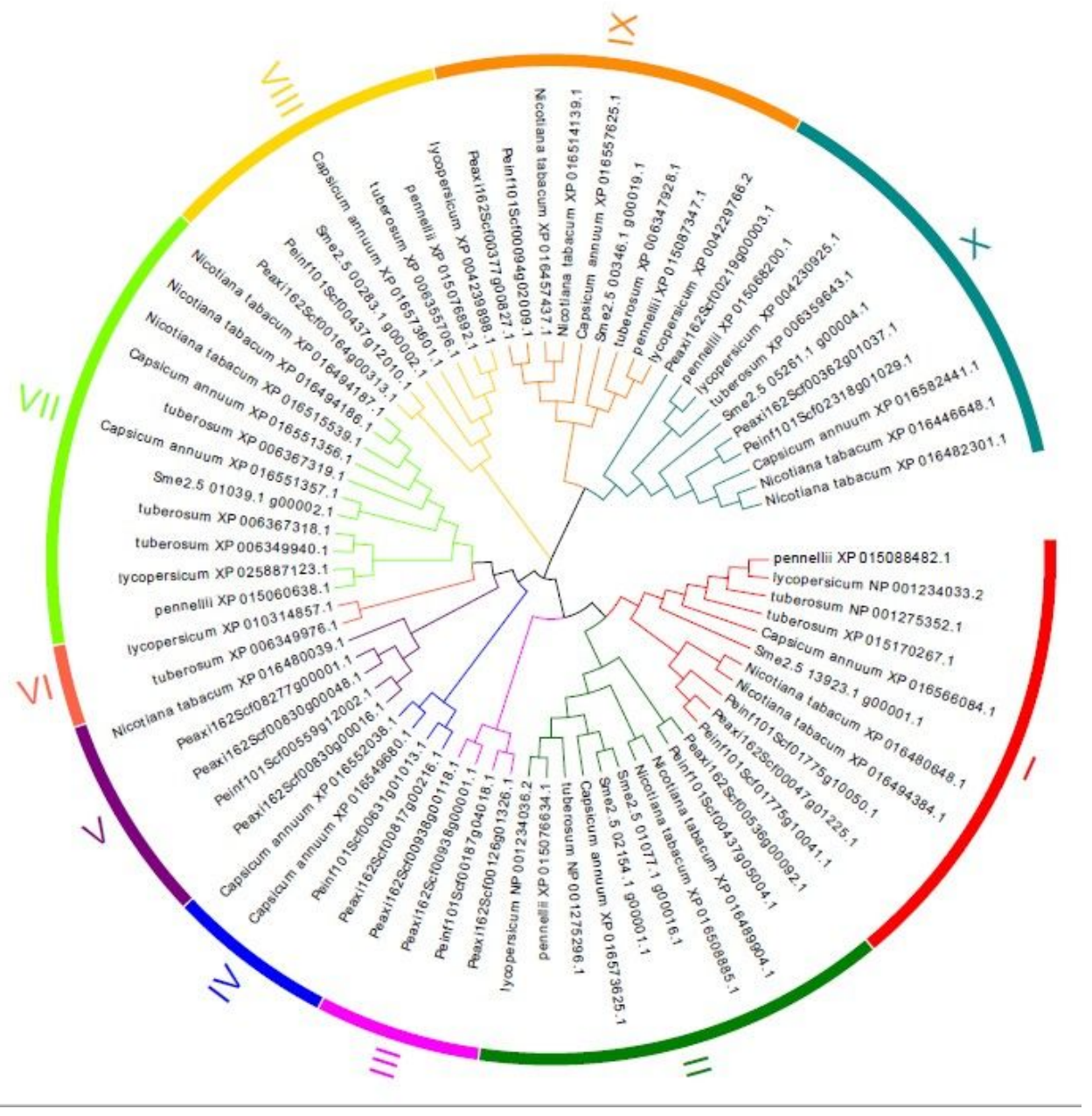

\section{Figure 4}

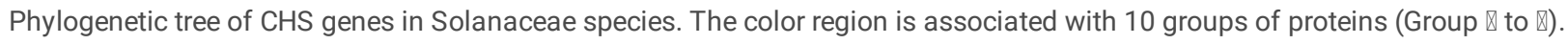




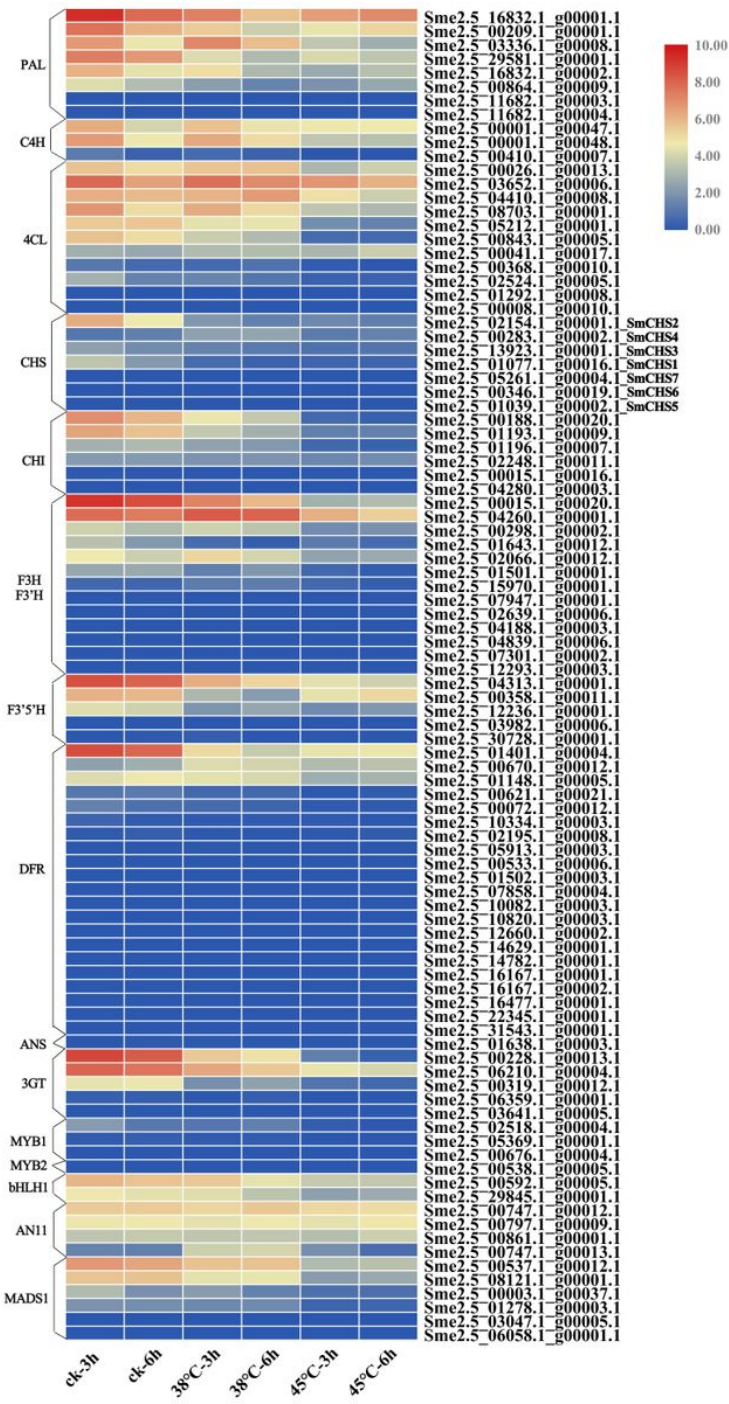

Figure 5

Expression profile of anthocyanin biosynthesis key genes in eggplant under heat stress. 


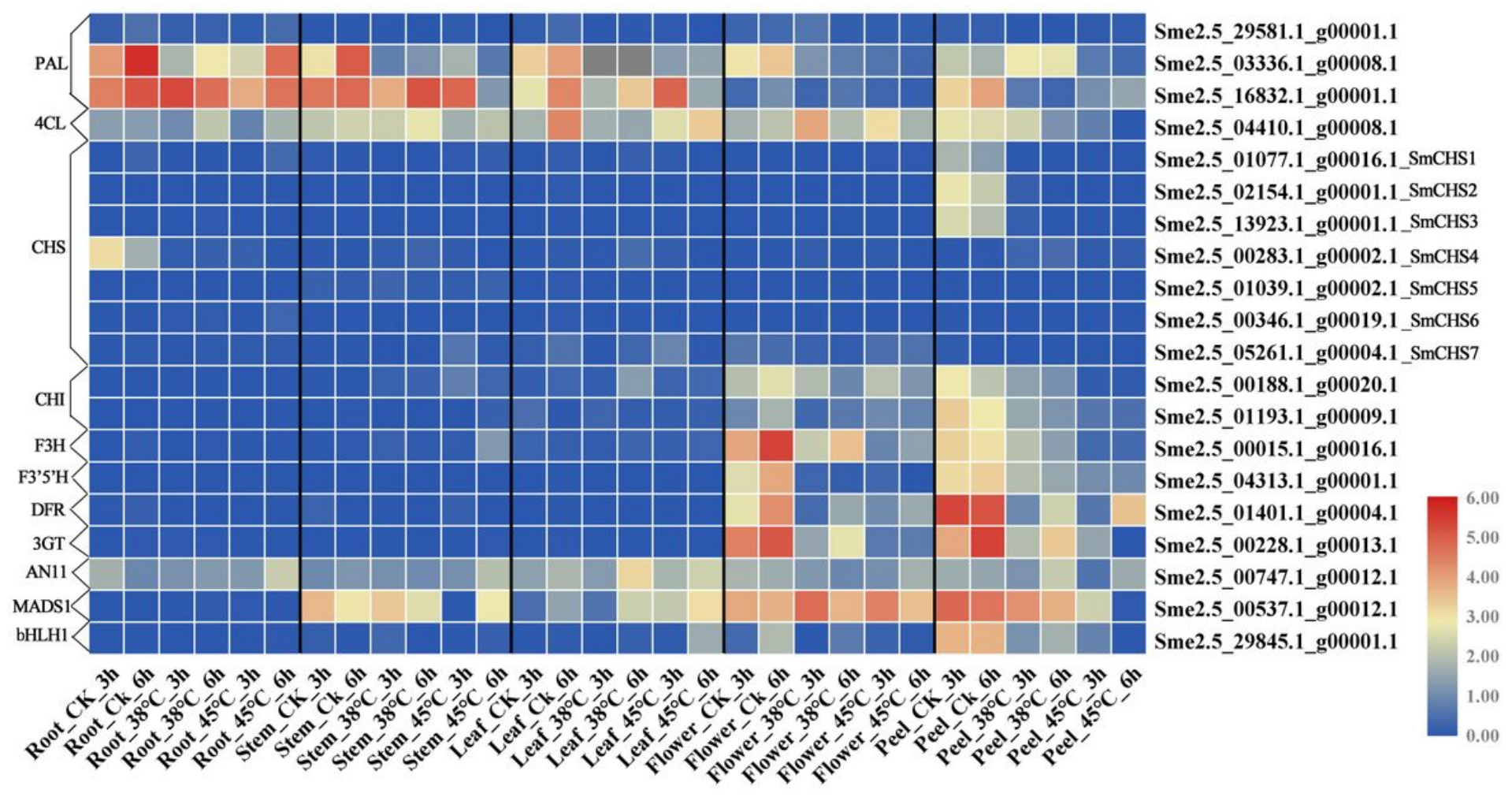

Figure 6

Expression profiles of 20 anthocyanin biosynthesis key genes in different tissues. 

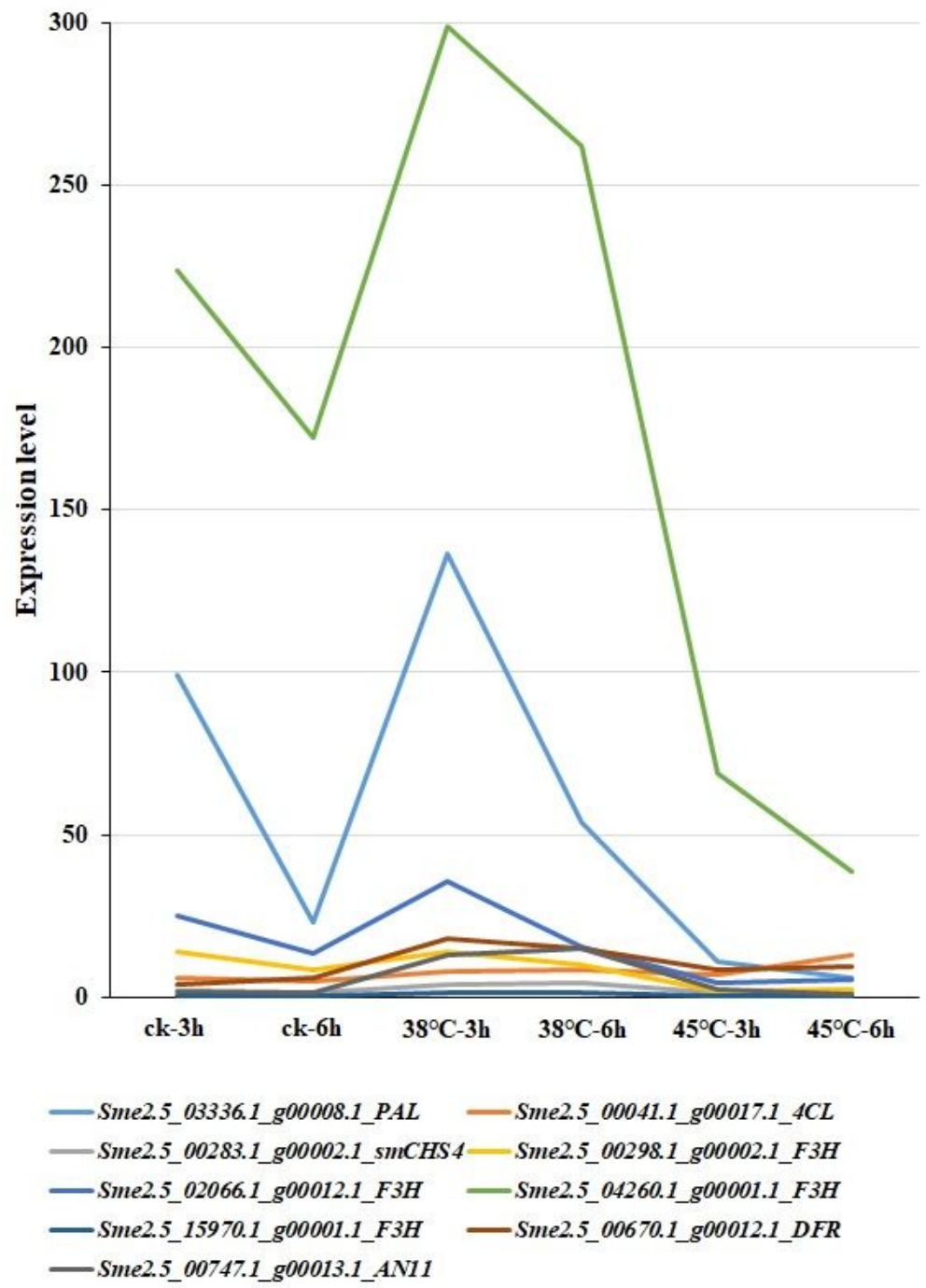

\section{Figure 7}

The anthocyanin biosynthesis key gene expression profiles in response to heat stress which were observed the highest expression level at $38^{\circ} \mathrm{C}-3 \mathrm{~h}$.

\section{Supplementary Files}

This is a list of supplementary files associated with this preprint. Click to download.

- Additionalfile3.docx

- Additionalfile1.xlsx

- Additionalfile4.pdf

- Additionalfile2.xlsx 